\title{
Aortopulmonary fistula in Friesian horses
}

Vercauteren Griet, Veronique Saey, Gunther van Loon, Marco De Bruijn, Catherine Delesalle and Koen Chiers

Faculty of Veterinary Medicine, Ghent University, Belgium griet.vercauteren@ugent.be

Introduction: Aortic rupture is very rare in animals. However, in Friesian horses this condition is more frequently encountered, suggesting this is a hereditary disorder. A congenital aneurysm, copper deficiency, long-term degenerative disease with weakening of the aorta, migration of Strongylus vulgaris and extreme hypertension in breeding stallions have been suggested. Here we describe 3 unusual manifestations of aortic rupture.

Materials and methods: Three friesian horses were submitted for necropsy with a suspicion of sudden aortopulmonary fistulation based on history, clinical signs, echocardiography and cardiac catheterisation. Histological examination of the aortic wall was performed.

Results: Grossly, the aortic wall was ruptured close to the ligamentum arteriosum with fistulation into the pulmonary artery. Perivascular hematomas were extensive. Histological examination revealed a thickening of the tunica media by increase of mucinous stroma. Focal areas were necrotic and occasionally thrombosis of vasa vasorum was present.

Conclusion: This defect could be associated with dysregulation of matrix metalloproteinases in Friesian horses. The cause of this condition is not known, although the presented histological lesions resemble cystic medial necrosis of the aorta, as seen in humans. 